\title{
GENERALIZED HIGHER DERIVATIONS
}

\author{
E. P. COJUHARI ${ }^{\bowtie}$ and B. J. GARDNER
}

(Received 13 October 2011)

\begin{abstract}
A type of generalized higher derivation consisting of a collection of self-mappings of a ring associated with a monoid, and here called a D-structure, is studied. Such structures were previously used to define various kinds of 'skew' or 'twisted' monoid rings. We show how certain gradings by monoids define $D$-structures. The monoid ring defined by such a structure corresponding to a group-grading is the variant of the group ring introduced by Năstăsescu, while in the case of a cyclic group of order two, the form of the $D$-structure itself yields some gradability criteria of Bakhturin and Parmenter. A partial description is obtained of the $D$-structures associated with infinite cyclic monoids.
\end{abstract}

2010 Mathematics subject classification: primary 13N15, 16S36, 16A03; secondary 16W55.

Keywords and phrases: derivation, higher derivation, graded ring, monoid algebra.

\section{Introduction}

All rings considered are associative with identity, and ring homomorphisms preserve identities.

A higher derivation of rank $m$ (respectively, of infinite rank) on a ring $R$ is a sequence $d_{0}, d_{1}, \ldots, d_{m}$ (respectively, $d_{0}, d_{1}, d_{2}, \ldots$ ) of additive endomorphisms of $R$ such that $d_{n}(a b)=\sum_{i+j=n} d_{i}(a) d_{j}(b)$ for each relevant $n$ and for all $a, b \in R$. The defining condition ensures that in both cases $d_{0}$ is an endomorphism and $d_{1}$ is a $\left(d_{0}, d_{0}\right)$-derivation (whence a derivation when $d_{0}$ is the identity map). This concept is quite well established and seems to have been introduced (for fields) by Hasse and Schmidt [4]. Higher derivations are closely related to homomorphisms from $R$ to $R[X] /\left(X^{m}\right)$ (in the rank- $m$ case) and to $R[[X]]$ (in the infinite-rank case) and (at least when $d_{0}$ is the identity) the $d_{i}$ can be described in terms of derivations $[1,5,6]$.

In [3] the first author introduced what we claim is a generalization of higher derivations. We first recall the definition and then give a justification for the claim.

Let $G$ be a monoid with identity $e, R$ a ring with identity 1 , and for each $x, y \in G$ let $\sigma_{x, y}: R \rightarrow R$ be a function. We require the set of $\sigma_{x, y}$ to satisfy the following condition, where $x, y, z$ are arbitrary elements of $G$ and $a, b$ of $R$.

(c) 2012 Australian Mathematical Publishing Association Inc. 0004-9727/2012 \$16.00 


\section{Condition A.}

(0) For each $x \in G$ and $a \in R$, we have $\sigma_{x, y}(a)=0$ for almost all $y \in G$.

(i) Each $\sigma_{x, y}$ is an additive endomorphism.

(ii) $\sigma_{x, y}(a b)=\sum_{z \in G} \sigma_{x, z}(a) \sigma_{z, y}(b)$.

(iii) $\sigma_{x y, z}=\sum_{u v=z} \sigma_{x, u} \circ \sigma_{y, v}$.

(iv $)_{1} \sigma_{x, y}(1)=0$ if $x \neq y$.

(iv $\left.{ }_{2}\right) \sigma_{x, x}(1)=1$.

(iv 3$) \sigma_{e, x}(a)=0$ if $x \neq e$.

(iv 4$) \sigma_{e, e}(a)=a$.

Definition 1.1. A collection of functions $\sigma_{x, y}$ satisfying Condition A is called a $D$ structure.

Condition A(iii) obviously has at least a superficial similarity to the defining condition for a higher derivation, but a closer examination reveals a more significant connection. Let $d_{0}, d_{1}, d_{2}, \ldots$ be a higher derivation of infinite rank in which for convenience we take $d_{0}$ to be the identity function. We make use of the additive monoid $\mathbb{N}$. For $x, y \in \mathbb{N}$ let $\sigma_{x, y}=(x ! / y !) d_{x-y}$ for $x \geq y$ and the zero function for $x<y$. We show that the $\sigma_{x, y}$ satisfy most of Condition A.

Clearly (i) is satisfied. If $x \geq y \in \mathbb{Z}^{+}$and $a, b \in R$, then

$$
\begin{aligned}
\sigma_{x, y}(a b) & =\frac{x !}{y !} d_{x-y}(a b)=\frac{x !}{y !} \sum_{t=0}^{x-y} d_{x-y-t}(a) d_{t}(b)=\frac{x !}{y !} \sum_{y \leq z \leq x} d_{x-z}(a) d_{z-y}(b) \\
& =\sum_{y \leq z \leq x} \frac{x !}{z !} d_{x-z}(a) \frac{z !}{y !} d_{z-y}(b)=\sum_{y \leq z \leq x} \sigma_{x, z}(a) \sigma_{z, y}(b),
\end{aligned}
$$

while for $x<y$ everything is zero, so we have (ii). It is easy to prove by induction that $d_{n}(1)=0$ for all $n \geq 1$ so (recalling that $d_{0}$ is the identity) for $x>y$ we have $\sigma_{x, y}(1)=(x ! / y !) d_{x-y}(1)=0$ while $\sigma_{x, x}(1)=(x ! / x !) d_{0}(1)=1$, whence $\left(\mathrm{iv}_{1}\right)$ and $\left(\mathrm{iv}_{2}\right)$ hold. Observing that $\sigma_{0, x}$ is zero for all $x>0$ and $\sigma_{0,0}=d_{0}$, we see that the rest of (iv) is valid too.

What about (iii)? A higher derivation $d_{0}, d_{1}, d_{2}, \ldots$ is iterative [4] if $d_{i} \circ d_{j}=$ $\left(\begin{array}{c}i+j \\ i\end{array}\right) d_{i+j}$ for each $i, j$. If (iii) is satisfied then, in particular,

so

$$
(i+j) ! d_{i+j}=\frac{(i+j) !}{0 !} d_{i+j}=\sigma_{i+j, 0}=\sigma_{i, 0} \circ \sigma_{j, 0}=i ! d_{i} \circ j ! d_{j},
$$

$$
d_{i} \circ d_{j}=\frac{(i+j) !}{i ! j !}=\left(\begin{array}{c}
i+j \\
i
\end{array}\right) d_{i+j}
$$

and thus the higher derivation is iterative. Conversely, if the higher derivation is iterative, then for each $x, y, z$ we have

$$
\begin{aligned}
\sum_{u+v=z} \sigma_{x, u} \circ \sigma_{y, v} & =\sum_{u+v=z} \frac{x !}{u !} d_{x-u} \frac{y !}{v !} d_{y-v} \\
& =\sum_{u+v=z} \frac{x !}{u !} \frac{y !}{v !}\left(\begin{array}{c}
x-u+y-v \\
x-u
\end{array}\right) d_{x-u+y-v}
\end{aligned}
$$




$$
\begin{aligned}
& =\sum_{u=0}^{z} \frac{x !}{u !} \frac{y !}{(z-u) !}\left(\begin{array}{c}
x+y-z \\
x-u
\end{array}\right) d_{x+y-z} \\
& =\sum_{u=0}^{z} \frac{z !}{u !(z-u) !} \frac{x ! y !}{z !}\left(\begin{array}{c}
x+y-z \\
x-u
\end{array}\right) d_{x+y-z} \\
& =\sum_{u=0}^{z}\left(\begin{array}{l}
z \\
u
\end{array}\right)\left(\begin{array}{c}
x+y-z \\
x-u
\end{array}\right) \frac{x ! y !}{z !} d_{x+y-z}=\left(\begin{array}{c}
x+y \\
x
\end{array}\right) \frac{x ! y !}{z !} d_{x+y-z} \\
& =\frac{(x+y) !}{x ! y !} \frac{x ! y !}{z !} d_{x-y+z}=\frac{(x+y) !}{z !} d_{x+y-z}=\sigma_{x+y, z},
\end{aligned}
$$

so (iii) is satisfied. Thus a higher derivation $d_{0}, d_{1}, d_{2}, \ldots$ defines a D-structure consisting of functions $\sigma_{x, y}=(x ! / y !) d_{x-y}$ for $x \geq y$ and zero otherwise if and only if the higher derivation is iterative. When there is a derivation $\delta$ such that $d_{n}=\delta^{n} / n$ ! for each $n$, the higher derivation $d_{0}, d_{1}, d_{2}, \ldots$ is iterative, and for $x \geq y$ we have

$$
\sigma_{x, y}=\frac{x !}{y !} \frac{\delta^{x-y}}{(x-y) !}=\left(\begin{array}{l}
x \\
y
\end{array}\right) \delta^{x-y} .
$$

This is the $D$-structure of [3, Example 2, pp. 31-32].

In [3] systems satisfying Condition A were used to construct 'skew' or 'twisted' monoid rings $R\langle G\rangle$ exemplified by various kinds of polynomial rings and Weyl algebras; (iii) is crucial for this construction.

In this paper our main concern is with the systems of functions themselves, though we briefly describe some associated monoid rings. We give some further examples of $D$-structures and, in particular, show how such systems can be defined by gradings of rings $R$ by certain monoids $G$. When the monoid is a group, the resulting monoid ring $R\langle G\rangle$, as constructed in [3], is the variant of the group ring introduced by Năstăsescu in [7]. In the special case where $G$ is a cyclic group of order two, we easily deduce from the form of the associated $D$-structure some criteria for $G$-gradability found by Bakhturin and Parmenter [2,8]. The possibility of characterizing other types of gradability by means of $D$-structure is worthy of exploration. Finally, we take the least complicated monoid, a singly generated one, which has a natural order, and examine in some detail those systems arising from this monoid in whose definition the order plays a special role.

Note that the connection between higher derivations and homomorphisms to polynomial and power series rings to which we referred earlier is mimicked by systems satisfying Condition A and matrix rings: the correspondence $A \mapsto\left(\sigma_{x, y}(a)\right)$ defines a homomorphism from $R$ to its ring of $|G| \times|G|$ matrices. We do not need (iii) for this. Thus systems satisfying Condition A correspond to homomorphisms to rings of matrices with structured label sets. 


\section{Gradings}

In this section we show that when a ring $A$ is graded by a monoid $G$ and satisfies a rather natural condition, (contravariant) actions of $G$ on monoids $H$ give rise to $D$ structures on $A$ defined by $H$. When $A$ is graded by $G$ we write $A=\sum_{g \in G} A_{g}$ and (often) denote an element of $A$ by notation such as $a=\sum_{g \in G} a_{g}$, where $a_{g}$ is the component of $a$ in $A_{g}$. For a monoid $H$ we denote by $E(H)$ the monoid under composition of all endomorphisms of $H$.

THeorem 2.1. Let $A$ be a ring with identity 1 graded by a multiplicatively written monoid $G$ with identity $e$ such that $1 \in A_{e}$. Let $\lambda: G \rightarrow E(H)$ be a monoid antihomomorphism for a monoid $H$. We write $\lambda_{g}$ instead of $\lambda(g)$ for $g \in G$. For each $x, y \in H$, let $\sigma_{x, y}(a)=\sum_{\lambda_{g}(x)=y} a_{g}$ for all $a=\sum_{g \in G} a_{g}$. The resulting functions form $a$ D-structure.

Notes.

(1) The right-hand-side sum in the definition of the $\sigma_{x, y}$ is defined even if $G$ is infinite: there are only finitely many nonzero $a_{g}$ anyway. If there are no relevant $a_{g}$ then the right-hand side is zero by a standard convention.

(2) If $G$ is cancellative or if $A$ is the monoid $\operatorname{ring} R[G]$ for some $\operatorname{ring} R$, then $1 \in A_{e}$ (though this condition is not satisfied by all graded rings.

Proof. We verify the requirements of Condition A. (0) and (i) are clear.

For $x, y \in H$ and $a, b \in A$,

$$
\begin{aligned}
\sigma_{x, y}(a b) & =\sigma_{x, y}\left(\sum_{w \in G} a_{w} \sum_{t \in G} b_{t}\right)=\sigma_{x, y}\left(\sum_{g \in G} \sum_{w t=g} a_{w} b_{t}\right) \\
& =\sum_{g \in G, \lambda_{g}(x)=y} \sum_{w t=g} a_{w} b_{t}=\sum_{y=\lambda_{w t}(x)} a_{w} b_{t}=\sum_{y=\lambda_{t}\left(\lambda_{w}(x)\right)} a_{w} b_{t} \\
& =\sum_{z=\lambda_{w}(x), y=\lambda_{t}(z)} a_{w} b_{t}=\sum_{z \in G}\left(\sum_{w \in G, z=\lambda_{w}(x)} a_{w} \sum_{y=\lambda_{t}(z)} b_{t}\right)=\sum_{z \in G} \sigma_{x, z}(a) \sigma_{z, y}(b),
\end{aligned}
$$

so (ii) is satisfied.

Now for $x, y, z \in H, a \in A$,

$$
\begin{aligned}
\sum_{u v=z} \sigma_{x, u} \circ \sigma_{y, v}(a) & =\sum_{u v=z} \sigma_{x, u}\left(\sigma_{y, v}(a)\right) \\
& =\sum_{u v=z} \sigma_{x, u}\left(\sum_{\lambda_{w}(y)=v} a_{w}\right) \sum_{u v=z}\left(\sum_{\lambda_{w}(y)=v} \sigma_{x, u}\left(a_{w}\right)\right) \\
& =\sum_{u v=z} \sum_{\lambda_{w}(y)=v, \lambda_{w}(x)=u} a_{w}=\sum_{\lambda_{w}(x) \lambda_{w}(y)=z} a_{w}=\sum_{\lambda_{w}(x y)=z} a_{w}=\sigma_{x y, z}(a) .
\end{aligned}
$$

This takes care of (iii).

Since $1=1_{e}$, for $x, y \in H$ we have $\sigma_{x, y}(1)=1$ if $y=\lambda_{e}(x)=x$ and 0 otherwise, so $\left(\mathrm{iv}_{1}\right)$ and $\left(\mathrm{iv}_{2}\right)$ hold. Finally, $\sigma_{e, e}(a)=\sum_{\lambda_{g}(e)=e} a_{g}=a$ for each $a \in A$, while if $x \neq e$, 
then $\sigma_{e, x}(a)=\sum_{\lambda_{g}(e)=x} a_{g}=0$ for each $a$. This gives us $\left(\mathrm{iv}_{3}\right)$ and $\left(\mathrm{iv}_{4}\right)$ and completes the proof.

Here are some illustrations of the theorem.

ExAmple 2.2. Take $G$ as a group, $H=G$ and for $g \in G$ let $\lambda_{g}(x)=g^{-1} x g$ for each $x \in G$. In this case the monoid algebra of [3] is Năstăsescu's graded group ring from [7].

Example 2.3. Let $G$ be a cancellative monoid and let $H=G \cup\{u\}, u \notin G$. Then $H$ is a monoid with respect to the operation $*$ which defines a right zero semigroup on $G$ and has $u$ as an identity, that is, $x * y=y$ for all $x, y \in G$ and $u * r=r=r * u$ for every $r \in H$. For $g \in G$ let $\lambda_{g}(x)=x g$ (monoid product) for all $x \in G$ and $\lambda_{g}(u)=u$. Then $\lambda_{g}$ is an endomorphism of $H$ since, for $x, y \in G$,

$$
\begin{gathered}
\lambda_{g}(x * y)=(x * y) g=y g=x g * y g=\lambda_{g}(x) * \lambda_{g}(y), \\
\lambda_{g}(x * u)=\lambda_{g}(x)=x g=x g * u=\lambda_{g}(x) * \lambda_{g}(u),
\end{gathered}
$$

and so on. Since, for $g, h \in G$, we have $\lambda_{g h}(x)=x g h=\lambda_{h}\left(\lambda_{g}(x)\right)$ for all $x \in G$ and $\lambda_{g h}(u)=u=\lambda_{h}(u)=\lambda_{h}\left(\lambda_{g}(u)\right)$, all conditions of the theorem are met. Now $\sigma_{x, y}(a)=$ $\sum_{x g=y} a_{g}$, and this is $a_{z}$ if there is a (necessarily unique) $z \in G$ with $x z=y$ and 0 in the contrary case. In particular, when $G$ is a group, $\sigma_{x, y}(a)=a_{x^{-1} y}$. We note for later reference that even if $G$ is not a group we always have $a_{g}=\sigma_{g, g^{2}}(a)$, so that our functions $\sigma_{x, y}$ can pick out each component of each member of $A$. Observe also that $\sigma_{x, u}$ is the zero function for each $x \in G$ (as well as $\sigma_{u, x}$, of course). Thus $u$ plays a rather passive role in the system.

We examine a special case: $G=\{e, x\}$ is a cyclic group of order two. Here the nonzero, nonidentity functions are as described:

$$
\sigma_{e, e}(a)=a_{e}, \quad \sigma_{e, x}(a)=a_{x}=\sigma_{x, e}(a) \quad\left(\text { as } x=x^{-1}\right), \quad \sigma_{x, x}(a)=a_{e},
$$

so each of them effectively determines the other. If A has characteristic 2 , then, for all $a, b \in A$,

$$
\begin{aligned}
\sigma_{e, x}(a) b+a \sigma_{e, x}(b) & =a_{x}\left(b_{e}+b_{x}\right)+\left(a_{e}+a_{x}\right) b_{x} \\
& =a_{x} b_{e}+a_{e} b_{x}+2 a_{x} b_{x}=a_{e} b_{x}+a_{x} b_{e}=\sigma_{e, x}(a b),
\end{aligned}
$$

that is, $\sigma_{e, x}$ is a derivation. Also $\sigma_{e, x}^{2}=\sigma_{e, x}$. This correspondence (reversible in fact) between $\mathbb{Z}_{2}$ gradings and idempotent derivations for rings of characteristic 2 was discovered by Bakhturin and Parmenter [8]. In the contrasting situation where $A$ is 2-torsion-free, let $f=\sigma_{e, e}-\sigma_{e, x}$. Then $f$ is an automorphism of order two and $a-f(a) \in 2 A$ (even without 2-torsion-freeness) and the (reversible) correspondence between such automorphisms and $\mathbb{Z}_{2}$ gradings in this case has been described in [2, Proposition 3.1].

Example 2.4. Let $G$ be a semilattice with identity $e$ and let $H$ be the semilattice obtained by the adjunction of another identity $u$ to $G$. For $g \in G$, let $\lambda_{g}(x)=x g$ for each 
$x \in G$ and $\lambda_{g}(u)=u$. Then $\lambda_{g}(x y)=x y g=x y g^{2}=x g y g=\lambda_{g}(x) \lambda_{g}(y)$ for all $x, y \in G$, $\lambda_{g}(x u)=\lambda_{g}(x)=x g=x g u=\lambda_{g}(x) \lambda_{g}(u)$, and so on, so that each $\lambda_{g} \in E(H)$. Clearly also $\lambda_{g h}=\lambda_{h} \circ \lambda_{g}$ for all $g, h \in G$. Thus, for any ring $R$, we can define a suitable family of $\sigma_{x, y}: H \rightarrow R[G]$ : in orthodox semigroup ring notation, $\sigma_{x, y}\left(\sum r_{g} g\right)=\sum_{x g=y} r_{g} g$. In terms of the natural order on $G, \sigma_{x, y}$ is zero unless $x \leq y$, and in the latter case $\sigma_{x, y}\left(\sum r_{g} g\right)=\sum_{x \vee g=y} r_{g} g$. When $G$ is linearly ordered, $\sigma_{x, y}\left(\sum r_{g} g\right)=r_{y} y$ when $x \leq y$ and 0 when $x>y$.

Example 2.5. Let $A$ be graded by the additive monoid $\mathbb{N}$ of nonnegative integers. Let $\langle Y\rangle$ denote the free monoid on a single generator $Y$. For each $n \in \mathbb{N}$ we get an endomorphism $\lambda_{n}$ of $\langle Y\rangle$ by requiring that $\lambda_{n}(Y)=Y^{2^{n}}$, and this leads to a homomorphism (and hence an antihomomorphism) $\lambda: \mathbb{N} \rightarrow E\left(\langle Y\rangle\right.$ ). We write $\sigma_{m, n}$ instead of $\sigma_{Y^{m}, Y^{n}}$ for members of the resulting system. We have $\sigma_{m, n}\left(\sum_{i \in \mathbb{N}} a_{i}\right)=$ $\sum_{\lambda_{i}\left(Y^{m}\right)=Y^{n}} a_{i}=\sum_{2^{i} m=n} a_{i}$ so $\sigma_{0,0}$ is the identity, $\sigma_{m, 2^{j} m}\left(\sum_{i \in \mathbb{N}} a_{i}\right)=a_{j}$ for all $m>0$, for all $j$, and all other $\sigma_{m, n}$ are zero. Observe that for each $m>0$ the sequence $\sigma_{m, m}, \sigma_{m, 2 m}, \sigma_{m, 4 m}, \ldots, \sigma_{m, 2^{n} m}, \ldots$ is a higher derivation. When $A=R[X]$, the monoid algebra construction of [3] produces a ring of polynomials over $R$ in two indeterminates $X, Y$ for which $Y X=X^{2} Y$.

\section{The case of a cyclic monoid}

It is natural that a comprehensive study of $D$-structures should begin with an investigation of those based on a cyclic monoid, and we shall here consider an infinite cyclic monoid, which is to say a free monoid $G$ on a single generator $x$ (so that the resulting monoid ring will be a polynomial ring in a single (generally noncentral) indeterminate. Being isomorphic to the nonnegative integers with addition, such a monoid carries a natural order. In [3], D-structures were considered for which $\sigma_{x, y}=0$ whenever $x>y$ (respectively, whenever $x<y$ ) with a restriction on the number of nonzero $\sigma_{x, x^{n}}$ (respectively, $\sigma_{x^{n}, x}$ ). We shall adhere to the notational practice introduced in [3] whereby $\sigma_{x^{n}, x^{m}}$ is called $\sigma_{n m}$ and, in particular, $\sigma_{e, e}$ is called $\sigma_{00}$.

For completeness we state two theorems from [3] with references for their proofs, but we have augmented the second with some parts which we prove below. Combinations of the following conditions on a $D$-structure are involved in these results.

(A') $\sigma_{n m}=0$ when $n<m$.

(A') $\sigma_{n m}=0$ when $n>m$.

(B) $\sigma_{11}$ is an automorphism.

(C) There is an $r$ such that $\sigma_{1 j}=0$ for every $j>r$.

$\left(\mathrm{C}^{\prime}\right)$ There is an $r$ such that $\sigma_{j 1}=0$ for every $j>r$.

(R) $\sigma_{11}, \sigma_{12}, \ldots, \sigma_{1 r}$ are right independent over $A$ ( $r$ as in $(\mathrm{C})$ ).

(L) $\sigma_{11}, \sigma_{21}, \ldots, \sigma_{r 1}$ are left independent over $A\left(r\right.$ as in $\left.\left(\mathrm{C}^{\prime}\right)\right)$. 
THEOREM 3.1. Under conditions $\left(A^{\prime \prime}\right),(B),(C)$ and $(R)$ the following assertions hold.

(i) The mapping

$$
\gamma_{0}:=\sigma_{12} \circ \sigma_{11}^{-1}
$$

is a $\left(1, \sigma_{11}\right)$-derivation of $A$.

(ii) $\gamma_{0}$ is a nilpotent mapping of index at most $r: \gamma_{0}^{r}=0$, and $\gamma_{0}^{r-1} \neq 0$ if $\sigma_{12} \neq 0$.

(iii) Each mapping $\sigma_{n m}(n \leq m)$ is expressible in terms of $\sigma_{11}$ and $\sigma_{12}$, namely,

$$
\begin{aligned}
\sigma_{n n} & =\sigma_{11}^{n} \quad(n=0,1, \ldots), \\
\sigma_{1 j} & =\gamma_{0}^{j-1} \circ \sigma_{11} \quad(j=1,2, \ldots, r), \\
\sigma_{n m} & =\sum_{j_{1}+\cdots+j_{n}=m} \sigma_{1 j_{1}} \circ \cdots \circ \sigma_{1 j_{n}}
\end{aligned}
$$

where $j_{k}=1,2, \ldots$ for $k=1, \ldots, n$.

The proof can be deduced from results in [3] as follows. (i) (6.20); (ii) Proposition 6.8; (iii) (6.19) and (6.35). Note that the third assertion in (iii) comes directly from Condition $\mathrm{A}$.

ReMark 3.2. From (1) we see that $\sigma_{n m}(n \leq m)$ is a sum of products of $\sigma_{11}$ and $\gamma_{0}$.

In the particular case when $\sigma_{11}$ and $\gamma_{0}$ commute, (3.1) gives us

$$
\sigma_{k j}=\left(\begin{array}{l}
j-1 \\
k-1
\end{array}\right) \sigma_{11}^{k} \circ \gamma_{0}^{j-k} \quad(k=1, \ldots, r, j=k, \ldots, r)
$$

and so, according to formulas (iii) and (iv) of Theorem 3.1,

$$
\sigma_{n r+k n r+j}=\left(\begin{array}{l}
j-1 \\
k-1
\end{array}\right) \sigma_{11}^{n r+k} \circ \gamma_{0}^{j-k} \quad(k=1, \ldots, r, j=k, \ldots, r)
$$

for $n=0,1,2, \ldots$.

THEOREM 3.3. Under conditions $\left(A^{\prime}\right),(B),(C)$ and $(L)$, the following hold.

(i) The mapping $\sigma_{10}$ is a $\left(\sigma_{11}, 1\right)$-derivation of $A$. In addition, $\sigma_{10}$ is a nilpotent mapping of index $r$,

$$
\sigma_{10}^{r}=0
$$

(ii) $\sigma_{n n}=\sigma_{11}^{n}, \sigma_{n 0}=\sigma_{10}^{n}$, for $n=0,1, \ldots$

(iii) The $\sigma_{k j} \quad(k=1, \ldots, r-1 ; j=0,1, \ldots, k)$ are sums of $\left(\begin{array}{l}k \\ j\end{array}\right)$ products of $j$ factors $\sigma_{11}$ and $k-j$ factors $\sigma_{10}$.

(iv) $\sigma_{r r} \circ \sigma_{j k}=\sigma_{j k} \circ \sigma_{r r}(j, k=0,1, \ldots)$.

(v) $\sigma_{10}^{n}(a b)=\sum_{j=0}^{n} \sigma_{n j}(a) \sigma_{10}^{j}(b)$ for all $a, b \in A, n=0,1, \ldots$ (generalized Leibniz rule).

(vi) The mapping

$$
\delta=\sigma_{11}^{-1} \circ \sigma_{10}
$$

is a $\left(1, \sigma_{11}^{-1}\right)$-derivation. Furthermore, $\delta^{r}=0$ and if $\sigma_{r-10} \neq 0$ then $\delta$ has index $r$. 
(vii) $\sigma_{k j}(k=1, \ldots, r-1 ; j=0,1, \ldots, k)$ is a sum of $\left(\begin{array}{l}k \\ j\end{array}\right)$ products of $k$ factors $\sigma_{11}$ and $k-j$ factors $\delta$.

The following parts of the theorem are proved in the places indicated in [3]. First assertion of (i) Proposition 6.1; (ii) (6.9), (6.8); (iii) follows from (6.7) by a straightforward counting argument.

For the rest of the proof we will need the following result.

Proposition 3.4. We have $\sigma_{k j}=0$ for $k=r, r+1, \ldots$, and $j=0,1, \ldots, r-1$.

Proof. The case $j=0$ is postulated by $\left(\mathrm{C}^{\prime}\right)$. For other values of $j$ the statement is implied by the property (L) of independence:

$$
\sigma_{k 0}(a b)=\sum_{j=0}^{k} \sigma_{k j}(a) \sigma_{j 0}(b) \quad(a, b \in A) .
$$

According to property $\left(\mathrm{C}^{\prime}\right)$, the last formula implies that, for $k \geq r$,

$$
\sum_{j=0}^{r-1} \sigma_{k j}(a) \sigma_{j 0}(b)=0 .
$$

Since the mappings $\sigma_{j 0}(j=0,1, \ldots, r-1)$ are $A$-independent from the left (property (L)), the formula (3.2) implies that

$$
\sigma_{k j}(a)=0 \quad(j=0,1, \ldots, r-1)
$$

for all $a \in A$.

Conclusion of proof of Theorem 3.3. Proposition 3.4 says that $\sigma_{r 0}=0$ and this, with (ii), implies that $\sigma_{10}^{r}=0$, which completes the proof of (i).

In fact, taken with our assumption (A'), Proposition 3.4 says that $\sigma_{r j}=0$ for all $j \neq r$, and then by (iii) of Condition A we have

$$
\sigma_{r+j r+k}=\sum_{u+v=r+k} \sigma_{r u} \circ \sigma_{j v}=\sigma_{r r} \circ \sigma_{j k}
$$

and

$$
\sigma_{j+r r+k}=\sum_{u+v=r+k} \sigma_{j u} \circ \sigma_{r v}=\sigma_{j k} \circ \sigma_{r r},
$$

for every $j, k=0,1, \ldots$, so (iv) is proved.

Concerning (vi), we first observe that, for all $a, b \in A$,

$$
\begin{aligned}
\delta(a b) & =\sigma_{11}^{-1}\left(\sigma_{10}(a b)\right)=\sigma_{11}^{-1}\left(\sigma_{10}(a) \sigma_{00}(b)+\sigma_{11}(a) \sigma_{10}(b)\right) \\
& =\sigma_{11}^{-1}\left(\sigma_{10}(a)\right) \sigma_{11}^{-1}\left(\sigma_{00}(b)\right)+\sigma_{11}^{-1}\left(\sigma_{11}(a)\right) \sigma_{11}^{-1}\left(\sigma_{10}(b)\right) \\
& =\left(\sigma_{11}^{-1} \circ \sigma_{10}\right)(a) \sigma_{11}^{-1}(b)+a\left(\sigma_{11}^{-1} \circ \sigma_{10}\right)(b) \\
& =\delta(a) \sigma_{11}^{-1}(b)+a \delta(b) .
\end{aligned}
$$


Thus $\delta$ is a $\left(1, \sigma_{11}^{-1}\right)$-derivation. Referring to Proposition 3.4 again, we see that, for $j=1, \ldots, r-1$,

$$
0=\sigma_{r j}=\sigma_{1+r-1 j}=\sum_{u+v=j} \sigma_{1 u} \circ \sigma_{r-1 v}=\sigma_{11} \circ \sigma_{r-1 j-1}+\sigma_{10} \circ \sigma_{r-1 j},
$$

whence

$$
\sigma_{r-1 j-1}=-\delta \circ \sigma_{r-1 j} \quad(j=1, \ldots, r-1) .
$$

Multiplying by $\delta$ from the left,

$$
\delta \circ \sigma_{r-1 j-1}=-\delta^{2} \circ \sigma_{r-1 j}
$$

and then, using (3.3),

$$
\sigma_{r-1 j-2}=\delta^{2} \circ \sigma_{r-1 j}
$$

for any $j=1, \ldots, r-1$. Repeating this procedure, we obtain the formula

$$
\sigma_{r-1 j-k}=(-1)^{k} \delta^{k} \circ \sigma_{r-1 j} \quad(j=1, \ldots, r-1 ; k=1, \ldots, j) .
$$

For $j=r-1$, formula (3.4) becomes

$$
\sigma_{r-1 r-1-k}=(-1)^{k} \delta^{k} \circ \sigma_{r-1 r-1} \quad(k=1, \ldots, r-1) .
$$

(see formula (8) in [9]).

By a change of variable we can rewrite (3.5) as

$$
\sigma_{r-1 k}=(-1)^{r-1-k} \delta^{r-1-k} \circ \sigma_{r-1 r-1} \quad(k=0,1, \ldots, r-1) .
$$

In particular, for $k=0$,

$$
\sigma_{r-10}=(-1)^{r-1} \delta^{r-1} \circ \sigma_{r-1 r-1}
$$

and if we compose on the left with $\delta$ and rearrange we get

$$
\begin{aligned}
\delta^{r} & =(-1)^{r-1} \delta \circ \sigma_{r-10} \circ \sigma_{r-1 r-1}^{-1} \\
& =(-1)^{r-1}\left(\sigma_{11}^{-1} \circ \sigma_{10}\right) \circ \sigma_{10}^{r-1} \circ \sigma_{r-1 r-1}^{-1} \\
& =(-1)^{r-1} \sigma_{11}^{-1} \circ \sigma_{10}^{r} \circ \sigma_{r-1 r-1}^{-1}
\end{aligned}
$$

and since $\sigma_{10}^{r}=0$,

$$
\delta^{r}=0 .
$$

There remains only (vii) and this follows from (iii) by the definition of $\delta$.

It is natural to ask whether there are converses of any kind for Theorems 3.1 and 3.3. In a sense there are in fact quite strong converses. If we take an arbitrary endomorphism (so not necessarily an automorphism) and a derivation of the type produced in Theorem 3.1, we can embed these two functions in a $D$-structure satisfying $\left(\mathrm{A}^{\prime \prime}\right)$ by imitating the structure determined by Theorem 3.1. In the same way, mutatis mutandis, we can get a $D$-structure satisfying $\left(\mathrm{A}^{\prime}\right)$ by mimicking the structure determined by Theorem 3.3. For these 'converses' we can dispense with $(C),\left(C^{\prime}\right),(R)$ and (L) completely. 
Theorem 3.5. For an endomorphism $\sigma_{11}$ and a $\left(1, \sigma_{11}\right)$-derivation $\gamma_{0}$ of a ring A, let $\sigma_{00}=1_{A}, \sigma_{0 n}=0$ for $n \geq 1, \sigma_{1 n}=\gamma_{0}^{n-1} \circ \sigma_{11}$ for $n \geq 1$. We define $\sigma_{n m}$ as in formula (i) of Theorem 3.1 for $1<n \leq m$ and $\sigma_{n m}=0$ for all $n>m$. The family thus defined determines a $D$ - structure on the ring $A$.

Proof. Since the mappings $\sigma_{11}$ and $\gamma_{0}$ are additive and, for $n \leq m, \sigma_{n m}$ are the sums of products (compositions of mappings) of the mappings $\sigma_{11}$ and $\gamma_{0}$, the mappings $\sigma_{n m}$ are also additive. So condition (i) of A holds.

As $\gamma_{0}$ is a $\left(1, \sigma_{11}\right)$-derivation of $A, \gamma_{0}(1)=0$. (Indeed, $\gamma_{0}(1)=\gamma_{0}(1 \cdot 1)=1 \gamma_{0}(1)+$ $\gamma_{0}(1) \sigma_{11}(1)$, where $\gamma_{0}(1)=\gamma_{0}(1)+\gamma_{0}(1)$ so, then, $\gamma_{0}(1)=0$.) In consequence, for $n \neq 1$,

$$
\sigma_{1 n}(1)=\left(\gamma_{0}^{n-1} \circ \sigma_{11}\right)(1)=\gamma_{0}^{n-1}\left(\sigma_{11}(1)\right)=\gamma_{0}^{n-1}(1)=0,
$$

so $\sigma_{1 n}(1)=0(n=2,3, \ldots)$. Then (3.1) implies that $\sigma_{n m}(1)=0$ for $n \neq m(n, m=$ $0,1, \ldots)$. Thus $\left(\mathrm{iv}_{1}\right)$ of Condition $\mathrm{A}$ is verified. The conditions $\left(\mathrm{iv}_{3}\right)$ and $\left(\mathrm{iv}_{4}\right)$ are true by definition. Also (iv 2 ) follows from

$$
\sigma_{n n}=\sigma_{11}^{n} \quad(n=1,2, \ldots),
$$

which is a particular case of the formula (3.1).

Thus we have to prove parts (ii) and (iii) of Condition A and we deal with (iii) first; in other words, we show that

$$
\sigma_{n+m l}=\sum_{u+v=l} \sigma_{n u} \circ \sigma_{m v}
$$

We have

$$
\sigma_{n+m l}=\sum_{j_{1}+\cdots+j_{n+m}=l} \sigma_{1 j_{1}} \circ \cdots \circ \sigma_{1 j_{n+m}},
$$

and on the other hand

$$
\begin{aligned}
\sum_{u+v=l} \sigma_{n u} \circ \sigma_{m v} & =\sum_{u+v=l}\left(\sum_{j_{1}+\cdots+j_{n}=u} \sigma_{1 j_{1}} \circ \cdots \circ \sigma_{1 j_{n}}\right) \circ\left(\sum_{k_{1}+\cdots+k_{m}=v} \sigma_{1 k_{1}} \circ \cdots \circ \sigma_{1 k_{m}}\right) \\
& =\sum_{u+v=l} \sum_{j_{1}+\cdots+j_{n}=u} \sum_{k_{1}+\cdots+k_{m}=v} \sigma_{1 j_{1}} \circ \cdots \circ \sigma_{1 j_{n}} \circ \sigma_{1 k_{1}} \circ \cdots \circ \sigma_{1 k_{m}} \\
& =\sum_{u+v=l} \sum_{j_{1}+\cdots+j_{n}=u k_{1}+\cdots+k_{m}=v} \circ \cdots \circ \sigma_{1 j_{n}} \circ \sigma_{1 k_{1}} \circ \cdots \circ \sigma_{1 k_{m}} \\
& =\sum_{j_{1}+\cdots+j_{n+m}=l} \sigma_{1 j_{1}} \circ \cdots \circ \sigma_{1 j_{n+m}}=\sigma_{n+m l} .
\end{aligned}
$$

(In the last sum we have made the substitutions $j_{n+1}=k_{1}, \ldots, j_{n+m}=k_{m}$.)

In the proof of (ii) the following formula will be useful:

$$
\sigma_{n+1 m+1}=\gamma_{0} \circ \sigma_{n+1 m}+\sigma_{11} \circ \sigma_{n m} \quad(n, m=1,2, \ldots) .
$$


We obtain (3.6) easily from

$$
\sigma_{n+1 m+1}=\sum_{j_{1}+\cdots+j_{n+1}=m+1} \sigma_{1 j_{1}} \circ \cdots \circ \sigma_{1 j_{n+1}}
$$

by sorting the terms which start with $\sigma_{11}$, that is, those of the form $\sigma_{11} \circ \sigma_{1 k_{1}} \circ \cdots \circ$ $\sigma_{1 k_{n}}$ where $k_{1}+\cdots+k_{n}=m$. We still have terms of the form $\sigma_{1 l_{1}+1} \circ \sigma_{1 l_{2}} \circ \cdots \circ$ $\sigma_{1 l_{n+1}}$ which according to

$$
\sigma_{1 l_{1}+1}=\gamma_{0}^{l_{1}} \circ \sigma_{11}=\gamma_{0} \circ\left(\gamma_{0}^{l_{1}-1} \circ \sigma_{11}\right)=\gamma_{0} \circ \sigma_{1 l_{1}},
$$

can be written as $\gamma_{0} \circ \sigma_{1 l_{1}} \circ \cdots \circ \sigma_{1 l_{n+1}}$, where $l_{1}+\cdots+l_{n+1}=m$. Thus

$$
\begin{aligned}
& \sigma_{n+1, m+1}=\sum_{k_{1}+\cdots+k_{n}=m} \sigma_{11} \circ \sigma_{1 k_{1}} \cdots \circ \sigma_{1 k_{n}}+\sum_{l_{1}+\cdots+l_{n+1}=m} \sigma_{1, l_{1}+1} \circ \sigma_{1 l_{2}} \cdots \circ \sigma_{1 l_{n+1}} \\
& =\sigma_{11} \circ \sum_{k_{1}+\cdots+k_{n}=m} \sigma_{1 k_{1}} \circ \cdots \circ \sigma_{1 k_{n}}+\gamma_{0} \circ \sum_{l_{1}+\cdots+l_{n+1}=m} \sigma_{1 l_{1}} \circ \sigma_{1 l_{2}} \circ \cdots \circ \sigma_{1 l_{n+1}} \\
& =\sigma_{11} \circ \sigma_{n m}+\gamma_{0} \circ \sigma_{n+1} m \text {. }
\end{aligned}
$$

Condition (ii), which in the case under consideration is represented by the formula

$$
\sigma_{n m}(a b)=\sum_{j=n}^{m} \sigma_{n j}(a) \sigma_{j m}(b) \quad(a, b \in A ; n \leq m, n, m,=0,1, \ldots)
$$

is verified by induction.

The cases $n=m=0$ and $n=m=1$ are trivial. For $n=1$ and $m=2$ we have to verify the formula

$$
\sigma_{12}(a b)=\sigma_{11}(a) \sigma_{12}(b)+\sigma_{12}(a) \sigma_{22}(b) \quad(a, b \in A) .
$$

This formula is verified directly:

$$
\begin{aligned}
\sigma_{12}(a b) & =\left(\gamma_{0} \circ \sigma_{11}\right)(a b)=\gamma_{0}\left(\sigma_{11}(a b)\right)=\gamma_{0}\left(\sigma_{11}(a) \sigma_{11}(b)\right) \\
& =\sigma_{11}(a) \gamma_{0}\left(\sigma_{11}(b)\right)+\gamma_{0}\left(\sigma_{11}(a)\right) \sigma_{11}\left(\sigma_{11}(b)\right) \\
& =\sigma_{11}(a)\left(\gamma_{0} \circ \sigma_{11}\right)(b)+\left(\gamma_{0} \circ \sigma_{11}\right)(a) \sigma_{11}^{2}(b) \\
& =\sigma_{11}(a) \sigma_{12}(b)+\sigma_{12}(a) \sigma_{22}(b)
\end{aligned}
$$

If now

$$
\sigma_{1 n}(a b)=\sum_{j=1}^{n} \sigma_{1 j}(a) \sigma_{j n}(b) \quad(a, b \in A)
$$


then

$$
\begin{aligned}
\sigma_{1 n+1}(a b)= & \left(\gamma_{0} \circ \sigma_{1 n}\right)(a b)=\gamma_{0}\left(\sigma_{1 n}(a b)\right) \\
= & \gamma_{0}\left(\sum_{j=1}^{n} \sigma_{1 j}(a) \sigma_{j n}(b)\right)=\sum_{j=1}^{n} \gamma_{0}\left(\sigma_{1 j}(a) \sigma_{j n}(b)\right) \\
= & \sum_{j=1}^{n}\left(\sigma_{1 j}(a) \gamma_{0}\left(\sigma_{j n}(b)\right)+\gamma_{0}\left(\sigma_{1 j}(a)\right) \sigma_{11}\left(\sigma_{j n}(a)\right)\right) \\
= & \sum_{j=1}^{n}\left(\sigma_{1 j}(a)\left(\gamma_{0} \circ \sigma_{j n}\right)(b)+\left(\gamma_{0} \circ \sigma_{1 j}\right)(a)\left(\sigma_{11} \circ \sigma_{j n}\right)(b)\right) \\
= & \sigma_{11}(a)\left(\gamma_{0} \circ \sigma_{1 n}\right)(b)+\sum_{j=2}^{n} \sigma_{1 j}(a)\left(\gamma_{0} \circ \sigma_{j n}\right)(b) \\
& \quad+\sum_{j=1}^{n-1}\left(\gamma_{0} \circ \sigma_{1 j}\right)(a)\left(\sigma_{11} \circ \sigma_{j n}\right)(b)+\left(\gamma_{0} \circ \sigma_{1 n}\right)(a)\left(\sigma_{11} \circ \sigma_{n n}\right)(b) .
\end{aligned}
$$

Since

$$
\gamma_{0} \circ \sigma_{1 j}=\sigma_{1, j+1} \quad(j=1,2, \ldots)
$$

and

$$
\sigma_{11} \circ \sigma_{n n}=\sigma_{n+1 n+1},
$$

changing the summing variable in the second sum, we have

$$
\begin{gathered}
\sigma_{1 n+1}(a b)=\sigma_{11}(a) \sigma_{1 n+1}(b)+\sum_{j=2}^{n} \sigma_{1 j}(a)\left(\gamma_{0} \circ \sigma_{j n}\right)(b) \\
+\sum_{j=2}^{n} \sigma_{1 j}(a)\left(\sigma_{11} \circ \sigma_{j-1 n}\right)(b)+\sigma_{1 n+1}(a) \sigma_{n+1 n+1}(b) \\
=\sigma_{11}(a) \sigma_{1 n+1}(b)+\sum_{j=2}^{n} \sigma_{1 j}(a)\left(\left(\gamma_{0} \circ \sigma_{j n}\right)(b)+\left(\sigma_{11} \circ \sigma_{j-1 n}\right)(b)\right) \\
+\sigma_{1 n+1}(a) \sigma_{n+1}(b) .
\end{gathered}
$$

According to formula (3.6),

$$
\gamma_{0} \circ \sigma_{j n}+\sigma_{11} \circ \sigma_{j-1 n}=\sigma_{j n+1} \quad(j=2,3, \ldots)
$$

and then

$$
\begin{aligned}
\sigma_{1 n+1}(a b) & =\sigma_{11}(a) \sigma_{1 n+1}(b)+\sum_{j=2}^{n} \sigma_{1 j}(a) \sigma_{j n+1}(b)+\sigma_{1 n+1}(a) \sigma_{n+1 n+1}(b) \\
& =\sum_{j=1}^{n+1} \sigma_{1 j}(a) \sigma_{j n+1}(b) .
\end{aligned}
$$


By the induction step we conclude that formula (3.8) is true for any natural number $n$.

Now we assume that formula (3.7) is true for any pair of natural numbers which do not exceed the numbers $n$ and $m$, respectively. Then by the formula (condition (iii) is already verified)

$$
\sigma_{n m}=\sum_{u+v=m} \sigma_{n-1 u} \circ \sigma_{1 v}
$$

we deduce that

$$
\begin{aligned}
\sigma_{n m}(a b) & =\sum_{u+v=m}\left(\sigma_{n-1} \circ \sigma_{1 v}\right)(a b)=\sum_{u+v=m} \sigma_{n-1}\left(\sigma_{1 v}(a b)\right) \\
& =\sum_{u+v=m} \sigma_{n-1} u\left(\sum_{j=1}^{v} \sigma_{1 j}(a) \sigma_{j v}(b)\right)=\sum_{u+v=m} \sum_{j=1}^{v} \sigma_{n-1}\left(\sigma_{1 j}(a) \sigma_{j v}(b)\right) \\
& =\sum_{u+v=m} \sum_{j=1}^{v} \sum_{k=n-1}^{u} \sigma_{n-1}\left(\sigma_{1 j}(a)\right) \sigma_{k u}\left(\sigma_{j v}(b)\right) \\
& =\sum_{u+v=m} \sum_{j=1}^{v} \sum_{k=n-1}^{u}\left(\sigma_{n-1 k} \circ \sigma_{1 j}\right)(a)\left(\sigma_{k u} \circ \sigma_{j v}\right)(b) \\
& =\sum_{u+v=m} \sum_{s=n}^{m} \sum_{k+j=s}\left(\sigma_{n-1 k} \circ \sigma_{1 j}\right)(a)\left(\sigma_{k u} \circ \sigma_{j v}\right)(b) \\
& =\sum_{s=n}^{m}\left(\sum_{k+j=s}\left(\sigma_{n-1} \circ \sigma_{1 j}\right)(a)\right)\left(\sum_{u+v=m}\left(\sigma_{k u} \circ \sigma_{j v}\right)(b)\right)=\sum_{s=n}^{m} \sigma_{n s}(a) \sigma_{s m}(b) .
\end{aligned}
$$

Therefore formula (3.7) is true for any pair of natural numbers which do not exceed the numbers $n$ and $m$. Thus (ii) of Condition A is verified.

THeORem 3.6. Let $\sigma_{11}$ be an endomorphism and $\sigma_{10}$ a $\left(\sigma_{11}, 1\right)$-derivation of a ring $A$.

Let $\sigma_{00}:=1_{A}, \sigma_{n m}=0$ for $n<m$, and for $n \geq m$ let

$$
\sigma_{n m}=\sum_{j_{1}+\cdots+j_{n}=m} \sigma_{1 j_{1}} \circ \cdots \circ \sigma_{1 j_{n}}
$$

where $j_{1}, \ldots, j_{n}$ take only values 0 or 1 and $m=0,1, \ldots$ (Note that in the right-hand side of (3.9) each product has $m$ factors $\sigma_{11}$ and $n-m$ factors $\sigma_{10}$, the total number of terms being $\left(\begin{array}{l}n \\ m\end{array}\right)$.) Then the $\sigma_{n m}$ form a D-structure.

Proof. Since $\sigma_{10}$ and $\sigma_{11}$ are additive, condition (i) is clear. As in the proof of Theorem 3.5, it will be easier to treat condition (iii) before (ii). Thus we first show that

$$
\sigma_{n+m l}=\sum_{u=0}^{l} \sigma_{n u} \circ \sigma_{m l-u} \quad(n+m \geq l ; l, n, m=0,1, \ldots) .
$$


We have

$$
\begin{aligned}
\sum_{u=0}^{l} \sigma_{n u} \circ \sigma_{m l-u} & =\sum_{u=0}^{l}\left(\sum_{j_{1}+\cdots+j_{n}=u} \sigma_{1 j_{1}} \circ \cdots \circ \sigma_{1 j_{n}}\right) \circ\left(\sum_{k_{1}+\cdots+k_{m}=l-u} \sigma_{1 k_{1}} \circ \cdots \circ \sigma_{1 k_{m}}\right) \\
& =\sum_{u=0}^{l} \sum_{j_{1}+\cdots+j_{n}=u} \sum_{k_{1}+\cdots+k_{m}=l-u} \sigma_{1 j_{1}} \circ \cdots \circ \sigma_{1 j_{n}} \circ \sigma_{1 k_{1}} \circ \cdots \circ \sigma_{1 k_{m}} .
\end{aligned}
$$

The number of terms in the last triple sum is equal to $\sum_{u=0}^{l}\left(\begin{array}{l}n \\ u\end{array}\right)\left(\begin{array}{c}m \\ l-u\end{array}\right)$, and since

$$
\sum_{u=0}^{l}\left(\begin{array}{l}
n \\
u
\end{array}\right)\left(\begin{array}{c}
m \\
l-u
\end{array}\right)=\left(\begin{array}{c}
n+m \\
l
\end{array}\right)
$$

(see the formulas in [3, Example 2.1], where the Weyl algebras are described), this number coincides with the number of terms in the sum of products which represents the decomposition of the mapping $\sigma_{n+m, l}$ in accordance with the formula (3.9). Therefore, denoting $j_{n+1}=k_{1}, \ldots, j_{n+m}=k_{m}$,

$$
\sum_{u=0}^{l} \sigma_{n u} \circ \sigma_{m l-u}=\sum_{j_{1}+\cdots+j_{n+m}=l} \sigma_{1 j_{1}} \circ \cdots \circ \sigma_{1 j_{n+m}}=\sigma_{n+m l},
$$

that is, formula (3.10).

Property (ii), that is, the formula

$$
\sigma_{n m}(a b)=\sum_{j=m}^{n} \sigma_{n j}(a) \sigma_{j m}(b) \quad(n \geq m, n=1,2, \ldots, m=0,1, \ldots)
$$

is deduced by induction with respect to $n$. For $n=1$ we have to show two cases, $m=0$ and $m=1$, and these are obvious because $\sigma_{10}$ is a $\left(\sigma_{11}, 1\right)$-derivation, $\sigma_{11}$ an endomorphism.

We assume that (3.11) is true for $n$, and $m$ is any number less than or equal to $n$. By (3.10), for any $m<n+1$,

$$
\sigma_{n+1 m}=\sigma_{n m-1} \circ \sigma_{11}+\sigma_{n m} \circ \sigma_{10} .
$$

Then, taking account of the inductive assumption,

$$
\begin{aligned}
\sigma_{n+1 m}(a b)= & \sigma_{n m-1}\left(\sigma_{11}(a b)\right)+\sigma_{n m}\left(\sigma_{10}(a b)\right) \\
= & \sigma_{n m-1}\left(\sigma_{11}(a) \sigma_{11}(b)\right)+\sigma_{n m}\left(\sigma_{11}(a) \sigma_{10}(b)+\sigma_{10}(a) b\right) \\
= & \sum_{j=m-1}^{n} \sigma_{n j}\left(\sigma_{11}(a)\right) \sigma_{j m-1}\left(\sigma_{11}(b)\right)+\sum_{k=m}^{n} \sigma_{n k}\left(\sigma_{11}(a)\right) \sigma_{k m}\left(\sigma_{10}(b)\right) \\
& \quad+\sum_{k=m}^{n} \sigma_{n k}\left(\sigma_{10}(a)\right) \sigma_{k m}(b)
\end{aligned}
$$


and then, grouping and using (3.12) again, we continue with

$$
\begin{aligned}
\sigma_{n+1}(a b)= & \sigma_{n m-1}\left(\sigma_{11}(a)\right) \sigma_{m-1 m-1}\left(\sigma_{11}(b)\right)+\sum_{k=m}^{n} \sigma_{n k}\left(\sigma_{11}(a)\right) \sigma_{k m-1}\left(\sigma_{11}(b)\right) \\
& +\sum_{k=m}^{n} \sigma_{n k}\left(\sigma_{11}(a)\right) \sigma_{k m}\left(\sigma_{10}(b)\right)+\sum_{k=m}^{n} \sigma_{n k}\left(\sigma_{10}(a)\right) \sigma_{k m}(b) \\
= & \left(\sigma_{n m-1} \circ \sigma_{11}\right)(a) \sigma_{m m}(b) \\
& +\sum_{k=m}^{n}\left(\sigma_{n k} \circ \sigma_{11}\right)(a)\left(\left(\sigma_{k m-1} \circ \sigma_{11}\right)(b)+\left(\sigma_{k m} \circ \sigma_{10}\right)(b)\right) \\
& +\sum_{k=m}^{n}\left(\sigma_{n k} \circ \sigma_{10}\right)(a) \sigma_{k m}(b) \\
= & \left.\sigma_{n}{ }_{m-1}^{n} \circ \sigma_{11}\right)(a) \sigma_{m m}(b) \\
& +\sum_{k=m}^{n}\left(\sigma_{n k} \circ \sigma_{11}\right)(a) \sigma_{k+1}(b)+\sum_{k=m}^{n}\left(\sigma_{n k} \circ \sigma_{10}\right)(a) \sigma_{k m}(b) .
\end{aligned}
$$

Now we write the first sum as a summation over $k-1$, and after grouping terms we use (3.12) yet again. We obtain

$$
\begin{aligned}
\sigma_{n+1 m}(a b)=( & \left.\sigma_{n m-1} \circ \sigma_{11}\right)(a) \sigma_{m m}(b)+\sum_{k=m+1}^{n+1}\left(\sigma_{n k-1} \circ \sigma_{11}\right)(a) \sigma_{k m}(b) \\
& \quad+\sum_{k=m}^{n}\left(\sigma_{n k} \circ \sigma_{10}\right)(a) \sigma_{k m}(b) \\
= & \left(\sigma_{n m-1} \circ \sigma_{11}\right)(a) \sigma_{m m}(b)+\left(\sigma_{n n} \circ \sigma_{11}\right)(a) \sigma_{n+1}(b) \\
& \quad+\sum_{k=m+1}^{n}\left(\sigma_{n k-1} \circ \sigma_{11}\right)(a) \sigma_{k m}(b) \\
& \quad+\sum_{k=m+1}^{n}\left(\sigma_{n k} \circ \sigma_{10}\right)(a) \sigma_{k m}(b)+\left(\sigma_{n m} \circ \sigma_{10}\right)(a) \sigma_{m m}(b) \\
= & \left(\left(\sigma_{n}{ }_{m-1} \circ \sigma_{11}\right)(a)+\left(\sigma_{n m} \circ \sigma_{10}\right)(a)\right) \sigma_{m m}(b)+\sigma_{n+1} n+1 \\
& \quad+\sum_{k=m+1}^{n}\left(\left(\sigma_{n k-1} \circ \sigma_{11}(a)+\left(\sigma_{n k} \circ \sigma_{10}\right)(a)\right) \sigma_{k m}(b)\right. \\
= & \sigma_{n+1}(b) \\
= & \sum_{k=m}^{n+1} \sigma_{n+1}(a) \sigma_{m m}(b)+\sigma_{n+1}(a) \sigma_{k m+1}(b) \sigma_{n+1}(b)+\sum_{k=m+1}^{n} \sigma_{n+1}(a) \sigma_{k m}(b)
\end{aligned}
$$

Thus, we obtain formula (3.11) for $n+1$ and $m=0,1, \ldots, n$. It is easy to prove that 
the formula is also true for $m=n+1$ :

$$
\sigma_{n+1 n+1}(a b)=\sigma_{n+1}(a) \sigma_{n+1}(b)=\sum_{k=n+1}^{n+1} \sigma_{n+1 k}(b) \sigma_{k n+1}(b) .
$$

Therefore formula (3.11) is true for any $n$ and any $m=0,1, \ldots, n$. Thus condition (ii) is shown.

It is trivial to check conditions (iv). Condition (iv $\left.)_{1}\right)$ is a consequence of the fact that $\sigma_{10}$ is a $\left(\sigma_{11}, 1\right)$-derivation and $\sigma_{11}(1)=1$ (the unity element 1 being a constant of derivation $\left.\sigma_{10}\right)$. The other conditions $\left(\mathrm{iv}_{2}\right),\left(\mathrm{iv}_{3}\right)$ and $\left(\mathrm{iv}_{4}\right)$ are fulfilled due to the definition of the mappings $\sigma_{n m}$.

We note that Example 2.5 provides examples of $D$-structures using an infinite cyclic monoid which are not covered by either of Theorems 3.5 and 3.6.

\section{References}

[1] S. Abu-Saymeh and M. Ikeda, 'On the higher derivations of communitive [sic] rings', Math. J. Okayama Univ. 29 (1987), 83-90.

[2] Yu. A Bahturin and M. M. Parmenter, 'Group gradings on integral group rings', in: Groups, Rings and Group Rings, Lecture Notes in Pure and Applied Mathematics, 248 (eds. A. Giambruno, C. Polcino Milies and S. K. Sehgal) (Chapman \& Hall/CRC, Boca Raton, FL, 2006), pp. 25-32.

[3] E. P. Cojuhari, 'Monoid algebras over noncommutative rings', Int. Electron. J. Algebra 2 (2007), 28-53.

[4] H. Hasse and F. K. Schmidt, 'Noch eine Begründung der Theorie der höheren Differentialquotienten einem algebraischen Funktionenkörper einer Unbestimmten', J. reine angew. Math. 177 (1937), 215-237.

[5] N. Heerema, 'Derivations and embeddings of a field in its power series ring', Proc. Amer. Math. Soc. 11 (1960), 188-194.

[6] J. B. Miller, 'Homomorphisms, higher derivations, and derivations', Acta Sci. Math. (Szeged) 28 (1967), 221-231.

[7] C. Năstăsescu, 'Group rings of graded rings. Applications', J. Pure Appl. Algebra 33 (1984), 313-335.

[8] M. M. Parmenter, personal communication.

[9] T. H. M. Smits, 'Nilpotent $S$-derivations', Indag. Math. 30 (1968), 72-86.

E. P. COJUHARI, Department of Mathematics, Technical University of Moldova, Ştefan cel Mare av. 168, Chişinău, MD-2004, Moldova

e-mail: ecojuhari@yahoo.com

B. J. GARDNER, Discipline of Mathematics, University of Tasmania, Private Bag 37, Hobart, Tas. 7001, Australia

e-mail: gardner@ hilbert.maths.utas.edu.au 